\title{
Integrated FLAG based Energy Efficient Routing with Drop Minimization in MANET
}

\author{
Arshee Khan \\ MTech Student \\ Computer Science \\ LNCT Bhopal
}

\author{
Sunil Phulre \\ Astt. Professor \\ Computer Science \\ LNCT Bhopal
}

\author{
Vineet Richariya, Ph.D \\ Head of Dept. \\ Computer Science \\ LNCT, Bhopal
}

\begin{abstract}
The conservation of energy and the improvement in lifetime of the network has been a challenging task in Mobile Ad hoc Networks (MANET) due to their dynamic and un-predictable behavior and topology change. A route selection and packet drop due to limited buffer(queue) size are two important factor that causes energy consumption by handling retransmission of dropped packets. So many energy aware routing and queue management strategies have been introduced individually. By combining energy based routing with queue management, the lifetime of the network can be increased most effectively. This paper proposes integrated maximum energy based routing with prediction of the route failure by energy estimation and Dynamic queue initialization to reduce packet drop. We modified AODV protocol and drop-tail queue. A Simulation has been performed on NS-2 with the parameter packet delivery fraction, Throughput, endto end delay and number of packets. By comparison planned approach with the old one better result has obtained.
\end{abstract}

\section{General Terms}

Energy based routing, Queue management, Energy consumption.

\section{Keywords}

MANET, AODV, PDF (packet delivery fraction), MAXEAODV.

\section{INTRODUCTION}

The wireless communication industry has seen tremendous growth in the last couple of years. The high availability of wireless networks and emergence of handheld computer, PDAs and Cell phones is now playing very important role in our daily routines. Today everyone wants to run an application on demand and anywhere. Surfing the internet from cars, railway station, airport, cafes, public locations, internet browsing on cell phones, and information or file exchange between devices without wires connectivity are just a few examples. All this ease is the result of mobility of wireless devices while being connected to a gateway to access the internet or information from the fixed or wired infrastructure (called Infrastructure based wireless network) or ability to develop an on demand, self organizing wireless network without relying on any available fixed infrastructure (Called Ad hoc networks). Fig 1 shows infrastructure less mobile Ad hoc Network.Taking energy into consideration, there are three approaches that are usually used to achieve the energy-efficiency in MANETs, 2005 [6]: Power-Control, Power-Save and Maximum-Lifetime rating. The MaximumLifetime routing approach looks for the nodes that have minimum energy so that they can be avoided from the path. In MANET each node acts as a router. All routers have some buffers at input and output port which holds the packets at the time of congestion. These buffers are maintained as a queue. Buffer (queue) overflow causes packet lost, long delay and larger overhead. A lifetime of the network can be increased by minimizing packet drop and delay. Because of its important role in routing, queue management techniques have become most challenging tasks for researcher now days.

Ad-hoc on-demand Distance Vector (AODV) is a routing protocol used for Mobile Ad-Hoc Networks (MANET) [1]. AODV is an on-demand routing protocol. It does not maintain routes for every node to every other node in the network. Whenever a route to the destination is needed, it initiates a route discovery process and the routes remains as long as they are necessary. AODV is loop free at all time. In the on demand protocols, routes are discovered between a source and a destination only when the need arises to send data. This provides a reduced overhead of communication and scalability.

AODV protocol does not consider the power usage of node it optimizes the routing with lowest delay. From the Perspective of energy, the shortest path is not always the optimal path. If the same paths are being utilized repeatedly due to the minimum number of routes, the nodes energy along these routes will be consumed quickly and they may exhaust batteries.

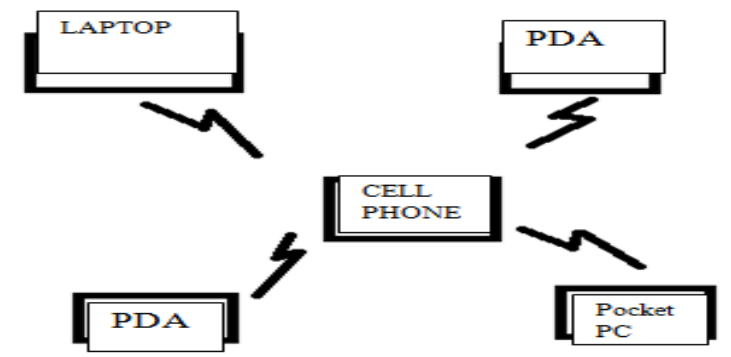

Figure 1 Mobile Ad Hoc Network

\section{Literature Survey}

Several researches have been proposed in the literature for the Energy based routing and Queue Management in Mobile Ad hoc Networks (MANET's). Some of them are as follows:

\section{The simulation and analysis of AODV routing protocol}

Some researchers [1] [2] found the AODV protocol to be the most popular MANET routing protocol, this has lead to many improvements being proposed by researchers to address some issues like end to end delay, reliability, route maintenance in wireless MANETs. 


\section{The Multiple Next Hops (MNH) routing} protocol [3] proposed by Jiang and Jan in 2001.According to the authors, the forward link and reverse link for each node may be used in AODV. For each destination, each source mobile node in MNH routing protocol maintains multiple next hops in its routing table. Hence the MNH may provide multiple routing paths for a source-destination pair. As link failure occurs, the upstream node will detect that and try to reconstruct a new route.

\section{Energy conserving routing in wireless} mobile ad hoc networks [4]. In 2000, the authors J.H. Chang, L. Tassiulas propose algorithms to select the routes and the corresponding power levels, such that the drain out time of the batteries of the nodes is until maximized. The uses to mode a single power level and multiple power levels to the maximum flow problem with node capacities. Then the achieved lifetime is close to the optimal computed by linear programming). The authors concluded with importance of balanced traffic load that in order to maximize the lifetime.The energy consumption of nodes in proportion to their energy reserves, instead of the routing to minimize the absolute consumed power.

\section{Minimum Battery Cost Routing (MBCR)} has been proposed in [5]. The residual energy of each node can be used as a quality route selection metric. To maximize reliability of transmission, the MBCR routing protocol calculates the sum of the residual energy of all nodes in an available paths and selects the higher energy content path. MBCR provides a route with higher energy, irrespective of every node minimum required residual power. So this method may choose a path containing mobile nodes with low energy. Thus, these low power mobile nodes may cause link breakage.

\section{Save power by using an efficient} broadcasting technique The researcher had pointed the effectiveness of efficient broadcasting techniques to save battery power. The broadcasting can be conducted using directional antennas for ad hoc networks. This paper firstly focuses on energy consumption as well as forwarding packets directionally by introducing directional antennas. Syropoulos et al [7], has implemented the use of Directional Antennas for energy efficient communication in ad hoc network.

\section{Krishna Cheong Lau and Joseph H. Kang}

[8] the idea to increase energy efficiency, nodes in the network goes into a sleep mode and wake up at predetermined time $\operatorname{slot}(\mathrm{s})$ to snoop for transmissions from its instant neighbors. The knowledge of awakening slots for neighboring nodes is used to arrange the transmissions within the neighborhood. Finally, nodes adapt their sleeping cycles based on neighbor topology and remaining battery life in order to maximize the network lifetime also satisfying the latency requirements of sensor applications.

Recently in 2012, A New Method for Restoration of Broken Links in Wireless Ad-hoc Networks by Estimation Energy Consumption has been introduced by Peyman Arebi [9]. This paper proposes a novel method based on energy estimation to restore broken links and reconstruct the paths of them. It reaches energy to a pre-determined level, it allows alarming. The determined level of declining energy alarm in various nods is different. This depends on the level of energy consumption in those nodes. In this study, regardless of hardware components influencing on energy consumption, nod's battery is considered as the basis of the residual energy determination. Analysis of packet loss due to queue with
bounded packet delay by $\mathrm{H}$. Schulzrinne, J. F. Kurose, and D. Towsley in 1990 [10]. For a buffer system emptying at a constant rate, an arrived packet is rejected if the sum of its length (in bits and the lengths of all buffered packets would overflow the buffer capacity. If it can be assumed that the service time of a packet depends only on its size and is thus known upon arrival, then the overall length of the buffered packets is bounded, and the model of a queue with bounded packet delay is more appropriate.

In 2007 Chen and Bensaou [11] presented a study for high speed networks about their survivability in terms of fairness and packet loss problems with Drop Tail queue management scheme. The authors mentioned when TCP flows come across multiple congested links in high speed networks working on Drop Tail scheme, they face packet drop probability unfairness and round trip time unfairness.

In 2009 Research [12] shown Drop Tail is a Passive Queue Management ( $\mathrm{PQM})$ algorithm which only sets a maximum length for each queue at the router. Routers decide when to drop packets. It uses first in, first out algorithm. In Drop Tail, the traffic is not differentiated. Each packet has the same priority. When the queue buffer is filled to its maximum capacity, the packets arrived afterward are dropped till the queue is full. That is, Drop Tail will keep discarding/dropping the packet until the queue has enough room for new packets.

\section{In 2010 Predictive queue management} strategy named PAQMAN [13] introduced by $\mathrm{K}$. Dinesh Kumar et cited that proactive management of the queue requires negligible computational overhead. PAQMAN does not require any prior knowledge of the traffic model, this reduces Packet loss ratio, Increases transmission efficiency. The performance has been compared with drop tail and those results show that PAQMAN reduces packet loss ratio while at the same time increasing transmission efficiency.

In 2012, Tolaimate Ichrak et al proposes the design of improved active Queue Management [14] control scheme for time delay systems using a time approach and synthesizing the linear fluid model. This method will reduce to delay through time approach. Obtaining a system delay free, the author applied to it the fundamentals of control theory as the similarity transformation, the pole placement by feedback, and by duality that construct an observer of the system. The resulting control laws are validated through numerical network estimations.

\section{PROPOSED WORK}

The proposed algorithm works in two different modules.

1. Maximum energy based routing with alarming signal for route failure-This module has enhanced the RREQ Handling process by considering node remaining energy as a routing metric. A route with maximum energy has chosen. Here predetermined threshold energy level is fixed. When a node reaches to this threshold value, new 
route computation gets started. This method works as an alarming signal to network link failure.

2. Dynamic Queue Size increment - The drop-tail queue has modified in this module. It has fixed queue limit. In modified module The simple drop-tail module drops the packet from the tail when it is overflowing. It transmits (dequeue) the packet in FIFO (First in First Out) manner. In modified module when a packet arrived for buffering, the queue size (byte) is calculated from-

$$
\text { qlimBytes }=\text { qlim_ } * \text { mean_pktsize_; }
$$

if occupied queue size with incoming packet size is greater then qlimBytes, the dynamic initialization of queues is performed for the new packet by incrementing queue limited use

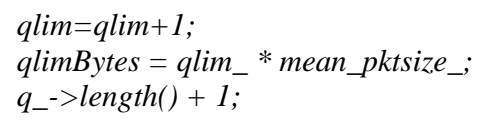

In above equation qlim is the instantaneous limit of the queue. mean_pktsize denotes size of the arrived packet.

\section{ALGORITHM (MAX-EAODV)}

1. Initialize E_threshold.// required minimum energy level.

2. Start route computation from source to destination d

Using step 3 and start buffering packet at the source.

3. Apply minimum threshold check on each node and

Select node with energy greater than E_threshold.

4. If node energy $<=E \_$threshold repeat step 2.

//Alarming condition for future link failure

Otherwise transmit packet through the maximum

Energy path.

5. If congestion or link failure occurs, go to step 6 .

6. If queue limit <=new required queue size go to step7

7. Initialize dynamic queue and increment queue limit by 1 .

// To reduce packet drop

8. Exit.

\section{SIMULATION}

For data collection and implementation, we will use Network Simulator- 2 (NS-2.31).
Table 1. Simulation parameter

\begin{tabular}{|l|l|}
\hline Simulation Parameter & Value \\
\hline Number of nodes & 40 \\
\hline Dimension of simulated area & $800 \times 800$ \\
\hline Routing Protocol & AODV \\
\hline Simulation time (seconds) & 100 \\
\hline Transport Layer & TCP ,UDP \\
\hline Traffic type & CBR , FTP \\
\hline Packet size (bytes) & 1000 \\
\hline Number of traffic connections & 10 \\
\hline
\end{tabular}

\section{PERFORMANCE PARAMETER}

1. Packet Delivery Fraction: The ratio of total data packets Successfully received to total ones sent by CBR source.

2. Remaining Energy: This refers to the difference between Total energy allotted to each node initially and total consumed energy of each node during complete simulation of $100 \mathrm{sec}$. This is important parameter of this research. The sum of energy remaining of each node will be shown as energy conservation by the network.

\section{RESULT ANALYSIS}

A comparison of the proposed approach with the old approach is given in figure 2. The $\mathrm{X}$-axis shows the interval of 20 seconds up to 100 seconds. The Y-axis shows a PDF value. The PDR_AODV is showing old AODV and PDR_MAX is showing proposed approach. Initially, both approaches start with approximate equal PDF value, but the graph of PDR_AODV gradually decreases as pause time increases from 0 to 20 seconds. The maximum $\operatorname{PDF}(96 \%)$ is $10 \%$ greater than $\mathrm{PDF}(86 \%)$ achieved in AODV between pause time 60 to 80 .The increased percentage of the PDF shows the least number of packets lost with respect to total transmitted packet.

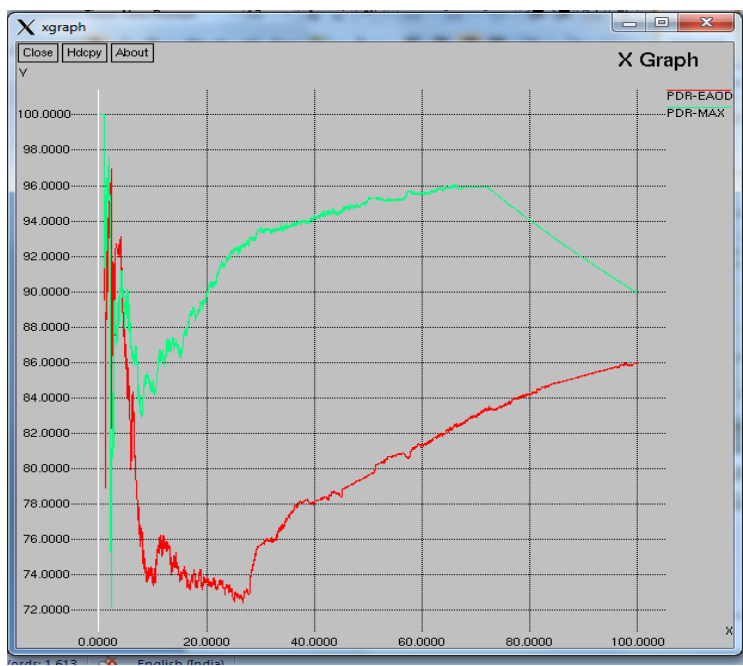

Fig 2 : Comparison graph of (PDF) 


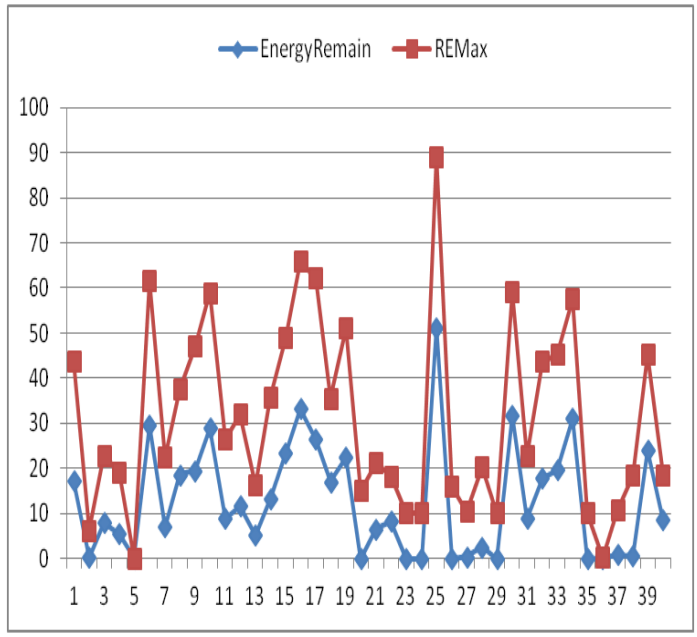

Fig 3 : Comparison graph of Remaining energy

An energy remained with each node has shown in figure 3.REMax is showing Proposed method.So it can be seen that energy remained by (737.84 joule) REMax is larger than old method EnergyRemain(507.01 joule).

Table 2. Overall analysis

\begin{tabular}{|l|l|l|}
\hline Parameter & AODV & $\begin{array}{l}\text { MAX- } \\
\text { EAODV }\end{array}$ \\
\hline Sent packet & 4643 & 5779 \\
\hline Received packet & 3986 & 5196 \\
\hline Routing packet & 4097 & 1435 \\
\hline Packet delivery fraction & $85.85 \%$ & $89.91 \%$ \\
\hline Drop data packet & 657 & 583 \\
\hline Drop routing packet & 169 & 18 \\
\hline Remaining Energy (joule) & 507.01 & 737.84 \\
\hline IFQ (dropped from queue) & 327 & 0 \\
\hline $\begin{array}{l}\text { Drop due to network } \\
\text { physical errors }\end{array}$ & 826 & 601 \\
\hline Total Drop & 1153 & 601 \\
\hline Performance & $\begin{array}{l}12704 \\
(91.68 \%)\end{array}$ & $12410(95.23 \%)$ \\
\hline
\end{tabular}

It is clear from table 2 that number of routing packet in AODV are much less as compare to MAX-EAODV.it shows less routing overhead in the network. Which in turn signals the less consumption of energy available in the network,tthi The parameters are calculated by following equations-

//Total number of data packet and routing packet(trans) consuming energy at the source node as well as at receiver node.

trans $=$ sends + recvs + routing packets ;

//Total number of packet drop due to interface queue and network congestion.here ifq denotes packet drop due to interface queue.

total_drop $=(i f q+$ Cong_Drop $)$;

//total represents total packet received successfully and dropped packet consuming energy. total $=$ trans + total_drop

//act_per denotes actual performance of the network.

act_per $=($ trans $* 100 /$ total $)$;

A number of packet drop due to interface queue in proposed approach is zero(0).A Large number of packet drop is minimized in turn conserve energy as well as increases network lifetime.

\section{CONCLUSION}

An Integrated FLAG based Energy efficient routing with drop minimization has been proposed. It works in two modules. It has been found from a simulation that proposed method outperforms the simple AODV protocol in terms of network lifetime, packet delivery ratio and packet loss. The overall performance has been improved by approximate $4 \%$. So it can provide excellent energy while at the same time achieving a much higher packet delivery ratio and lower packet loss than the existing protocol. In the future this can be extended to other routing protocols.

\section{REFERENCES}

[1] M. Morshed, H. Rahman, R. R. Mazumder, and K. A. M. Lutfullah "Simulation and Analysis of Ad-hoc Ondemand Distance Vector Routing Protocol," in Proc. of ICIS, November 24-26, 2009 Seoul, Korea, pp. 610-614.

[2] A. Boukerche et al., "Routing protocols in ad hoc networks: A survey," Computer Networks: The International Journal of Computer and Telecommunications Networking, vol. 55, no. 13. pp. 3032-3080, May2011.

[3] M.H. Jiang, R.H. Jan, An efficient multiple paths routing protocol for ad-hoc networks, in: Proceedings of Fifteenth IEEE International Conference on Information Networking, pp.544-549. 2001.

[4] J.H. Chang, L. Tassiulas, Energy conserving routing in wireless ad hoc networks, in: Proc. INFOCOM'00, IEEE, pp. 22-31, 2000.

[5] S. Singh, M.Woo and C.S. Raghavendra, "Power-Aware Routing in Mobile Ad hoc Networks,", MobiCom '98 Proceedings of the 4th annual ACM/IEEE, volume-1, pp181-190, 1998.

[6] J. Li, D. Cordes, and J. Zhang, "Power-aware routing protocols in ad hoc wireless networks," IEEE Trans. Wireless Commun., pp. 69-81, Dec. 2005

[7] Spyropoulos,Raghavendra," Energy efficient communications in ad hoc networks using directional antennas", C.S. INFOCOM Twenty-First Annual Joint Conference of the IEEE Computer and Communications Societies. Proceedings. IEEE, pp 220 - 228 vol.1,2002.

[8] Krishna Balachandran, Joseph H. Kang, Wing Cheong Lau: Adaptive sleeping and awakening protocol (ASAP) for energy efficient adhoc sensor networks. ICC 2005: 1068-1074.

[9] Peyman Arebi," A New Method for Restoration of Broken Links in Wireless Ad-hoc Networks by EstimationEnergyConsumption",IEEECommunicat on Systems and Networks conference,pp.377-381,2012. 
[10] H. Schulzrinne, J. F. Kurose, and D. Towsley, "Congestion control for real-time traffic in high-speed networks," IEEE Infocom, San Francisco,CA, pp: 543550, June 1990.

[11] K.Dinesh Kumar, I.Ramya \& M.Roberts Masillamani, "Queue Management in Mobile Adhoc Networks (Manets)" 2010 IEEE.
[12] Zhenyu Na and Qing Guo "An Improved AQM Scheme with Adaptive Reference Queue Threshold" 978-1-4577, 2011, IEEE

[13] Tolaimate Ichrak and Elalami Nourredine "Basic control theory Applied to the Active Queue Management model" 978-1-4673 1520, 2012, IEEE 